\title{
Application of Array Pressure Sensor in Roller Fault Detection
}

 \\ Leyong $\mathrm{Yu}^{2, \mathrm{f}}$, Yun $\mathrm{Hu}^{2, \mathrm{~g}}$, Dapeng Wei ${ }^{1,2, \mathrm{~h}}$ \\ ${ }^{1}$ School of Computer Science and Technology, Chongqing University of Posts and \\ Telecommunications, Chongqing 400065, China;
}

${ }^{2}$ Chongqing Institute of Green and Intelligent Technology, Chinese Academy of Sciences, Chongqing 400714, China.

aqyukun1992@163.com; bsallyyinsr@qq.com; *, c jyang@cigit.ac.cn; d liuht@cqut.edu.cn; etsun@cigit.ac.cn; 'lyyu@cigit.ac.cn; 9yhu@cigit.ac.cn; hdpwei@cigit.ac.cn

Keywords: Array pressure sensor; Fault Diagnosis; Neural Network; Probabilistic Neural Network (PNN); Roller group.

\begin{abstract}
In order to diagnose the fault of roller group, we have designed an array pressure sensor and a scan circuit with weak crosstalk of the column signal for collecting pressure distribution data. Furthermore, these data are processed via both the probabilistic neural network (PNN) fault diagnosis model and the back propagation (BP) neural network fault diagnosis model. Experimental results reveal that the PNN fault diagnosis model has more accuracy and shorter period of constructing and training model than BP fault diagnosis model. Our research opens up a new way for roller fault detection from via indirect information (such as sound, vibration and other signals) to via direct digitization pressure image. This method reduces the interference of environmental noise on fault detection. It is more accurate, intuitive, and potential for the applications in all kinds of mechanical fault detection.
\end{abstract}

\section{Introduction}

In industrial production, it is very important to detect equipment failure in timely, efficiently and accurately. The quality of product, such as lamination machine, grinding machine, printing press and so on, principally depends on the roller group which is a common equipment parts in the industrial production, in which the fault detection of the roller wheel is particularly important.

At present, the research of fault detection methods for machinery and equipment components mainly extracts fault feature data from voice and vibration signals which are used to detect faults by the signal processing method [1][2]. Signals of sound and vibration are widely used in the state of the equipment failure detection and judgment. However, the sound signal is susceptible to the surrounding environment, and contains a lot of noise; the non-linear and non-stationary signal processing method cannot accurately reflect the essential characteristics of the vibration signal because of its characteristics of weakness, non-linearity and non-stationaries [3]. Therefore, the above methods are not suitable for detecting the fault of the roll wheel group. With the development of fault diagnosis, there are a variety of algorithms for fault diagnosis, such as fault diagnosis based on casebased method, expert system, fuzzy reasoning, the BP neural network, and the pattern recognition fault diagnosis method. Among these, the method based on BP neural network is the most widely used [4]. However, there remains some problems, such as low rate of convergence and the problem of falling into local minimum, which cannot satisfy the requirement of high real-time and high stability to the system.

To detect the defect of roller group, we designed a new kind of flexible array pressure sensor to collect the pressure distribution, which act as the data of fault detection, between the roller groups. In view of the advantages that the probabilistic neural network (PNN) has such as easy to train, fast convergence speed, good expandability, robust classification ability without local minima problem and easily implemented in hardware [5], we choose PNN to train the model to detect the fault of roller wheels with avoiding the interference of environmental signal noise on data acquisition. 
In this paper, a lamination machine is acted as an example to study the application of the array pressure sensor in the fault detection of the roller group.

\section{The Design of Hardware Part}

\subsection{The Design of the Array Pressure Sensor}

A flexible array pressure sensor is a sensor that multiple pressure sensors are integrated in a plane to sense the pressure distribution. Flexible array pressure sensor is a resistive sensor and its working principle is as same as the general resistive sensor, that the contact force is acted on the force sensitive element which transforms the physical quantity into a resistance change. The force information is finally obtained by the inverter with transforming the change of voltage [6]. At present, many companies are committed to the research of flexible array pressure sensor, such as I-Motion, Tekscan, etc. The structure of the array pressure sensor is relatively simple in which the force-sensitive materials is printed on row and column electrodes, and two sensitive films are respectively attached together, then each column and row electrode is crossed and the crossed part was used as a sensor unit. The surface resistance of the force-sensitive material is very large and approximately insulated. When force is applied on the array pressure sensor, the bigger the contact area of the sensor is, the smaller the resistance is. This sensor structure is relatively simple and easy to produce, but the crosstalk noise from the column and row force-sensitive unit is large and it would have a great influence on the data of force distribution.

A new type of array of pressure sensor was designed that it has three layers, as shown in Figure 1 (a). The bottom layer is a flexible FPC circuit with a brush finger electrode. The top layer is a PET film with a force sensitive layer unit and the middle is coated with OCA adhesive. The sensor is 16 (row) $\times 16$ (column). The array unit size is $5 \mathrm{~mm} \times 5 \mathrm{~mm}$, and the sensor area is $127 \mathrm{~mm} \times 127 \mathrm{~mm}$. The flexible FPC circuit connects the individual units in a determinant. The photograph of the array pressure sensor is shown in Figure 1 (b).
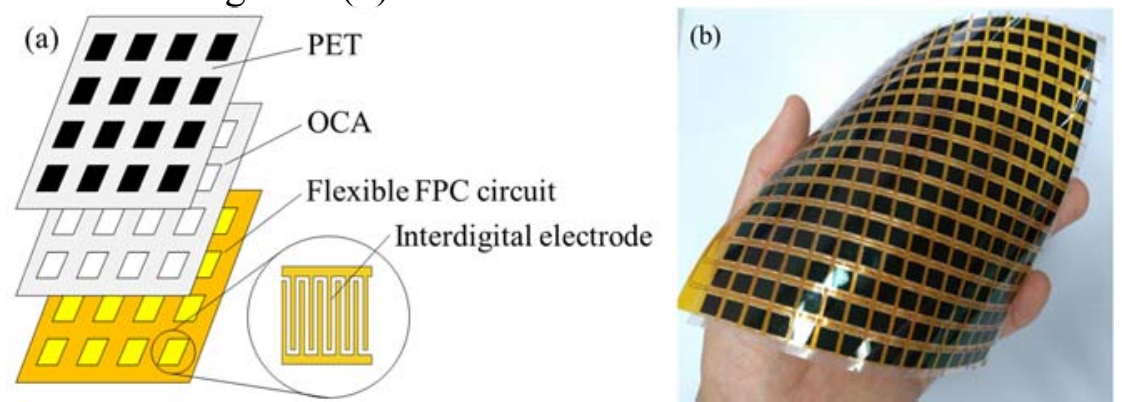

Fig. 1 Structure of Array Pressure Sensor. (a) Structure diagram; (b) Physical map.

For a single sensor cell, the sensitive layer is separated from the electrode at zero pressure with infinite resistance. When the force is applied to the sensor surface, the force-sensitive layer contacts the electrode. As the pressure increases, the resistance of the force-sensing sensor unit becomes smaller. The pressure-resistance (F-R) curve of a single sensor unit is shown in Figure 2. In order to illustrate the reliability of the sensor, the single-point test of the sensor and I-Motion array pressure sensor in this study is shown in Figure 3 (a) and Figure 3 (b).

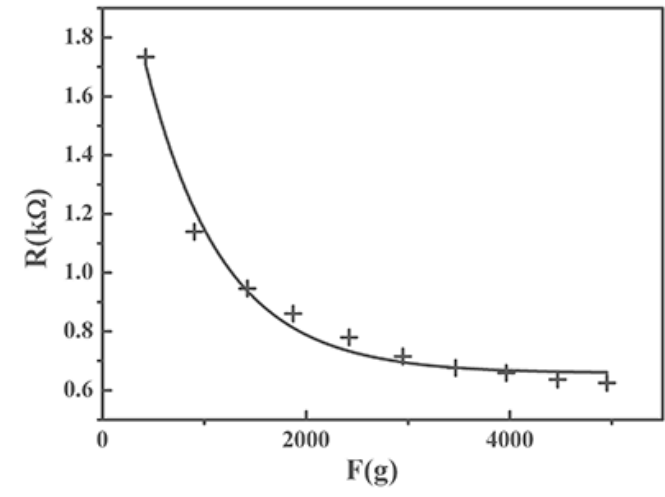

Fig. 2 Pressure sensor unit F-R curve 



Fig. 3 Array pressure sensor single point pressure test. (a) Single point test. (b) Single point test of I-Motion.

It was observed that the crosstalk noise from I-Motion array pressure sensor is more serious. The array pressure sensors in the study has been greatly improved on the crosstalk problem, it can reduce the collection between the upper and lower roller pressure data error.

\subsection{The Design of the Data Acquisition System}

The microcontroller uses the STM32F103RT in a data acquisition system that uses a highperformance ARM Cortex-M3 32-bit RISC core with an operating frequency of $72 \mathrm{MHz}$, built-in high-speed memory (up to $128 \mathrm{~K}$ bytes of flash and $20 \mathrm{~K}$ bytes of SRAM), rich I/O ports and peripherals connected to two APB buses, including two 12-bit ADCs, three general-purpose 16-bit timers and one PWM timer, a standard and advanced communication interface 2 I2C and SPI, 3 USARTs, one USB and one CAN. It can guarantee all the resources were used by the system.

The data acquisition system consists of array sensor, control circuit and PC and the block diagram, which is shown in Figure 4. The row of the array sensor is connected to the row select multiplexer, the strobe line chooses $5 \mathrm{~V}$ voltage, not strobe line chooses zero voltage, the column direction is connected to the "-" pole of the op amp, and the "+" pole of the op amp is connected to the ground. According to the nature of the op amp, the potential of each column is "virtual ground" [8]. This is done to avoid the crosstalk noise between the ranks. When the resistance of the sensor was changing, the output voltage of the op amp would also change.



Fig. 4 Data acquisition system structure diagram

For a single sensor unit, the circuit diagram was shown in Figure 5. According to the nature of the op amp, the formula is as follows

$$
V_{\text {out }}=-\frac{R_{f}}{R} \times V_{c c}
$$

It can be easily calculated corresponding to the sensor unit resistance, thus calculating the sensor surface pressure. 




Fig. 5 The circuit diagram of the sensor unit

The software design of data acquisition and processing system is consisted of lower computer for collecting and sending data, and upper computer for receiving and displaying. In host computer, MATLAB is used for graphical programming because MATLAB has a powerful data processing capability, image processing capabilities and a large number of functions for advanced data processing. Firstly, the system starts running and the upper computer and lower computer is initialized. Then, upper computer sends the transformation instruction to the lower computer according to communication protocols. The lower computer receives the transformation instruction and parses instruction. The lower computer begins to scan the lines and reads the converted voltage data. Then the voltage data is converted into pressure data which is send to the upper computer according to communication protocols. The upper computer saves the pressure data into buffer. If buffer read the data which is conformed to object format, it will parse and process data. Then the data is converted into pressure value and the pressure distribution and judgement are displayed.

\section{Fault Detection Algorithm}

The fault detection algorithm is mainly reflected to classify the fault data according to the fault model. In this study, the probabilistic neural network is used to detect the fault of roller group.

\subsection{Probabilistic Neural Network}

The Probabilistic Neural Network (PNN) is a simple and widely proposed by Dr. D.F.Specht in 1989, which is a parallel computing method based on Bayes classification rule and parzen window probabilistic density function estimation method [9]. The probabilistic neural network can use the linear learning algorithm to realize the function of nonlinear learning algorithm, which is regarded as a radial basis function neural network. Compared with BP neural network, PNN is a local approximation network. For each training sample, only a small number of weights and thresholds are needed to correct. Therefore, the training and learning speed of the network are fast and can be applied to some backgrounds which required strong real-time [10].

Probabilistic neural network has four layers, respectively input layer, hidden layer, sum layer and output layer. The structure is shown in Figure 6.



Fig. 6 The Probabilistic Neural Network structure

The first layer is the input layer, which is used to receive the training samples. The number of input neurons is the same as the input vector length.

The second layer is the implicit layer (The distance between the input vector and the center), which receives the sample input of the input layer. Each neuron in the hidden layer has a center, and the number of neurons is the same as the number of input samples. The vector $\mathrm{x}$ is input to the hidden layer. The relation between input and output of neuron $\mathrm{j}$ in implicit layer is defined as [11]: 


$$
\Phi_{i j}(\boldsymbol{x})=\frac{1}{(2 \pi)^{\frac{1}{2}} \sigma^{d}} e^{-\frac{\left(x-x_{i j}\right)\left(x-x_{i j}\right)^{T}}{\sigma^{2}}}
$$

Where $\mathrm{i}=1,2,3, \cdots, \mathrm{M}, \mathrm{M}$ is the number of training sample categories, $\mathrm{j}=1,2,3, \cdots \mathrm{L}, \mathrm{L}$ is the number of samples. $x i j$ is the $\mathrm{j}$ center vector of the $\mathrm{i}$ class. The $\sigma$ is the smoothing factor which determines the width of the bell-shaped curve centered on the sample point.

The sum of the neurons is the same as the number of classes, and the summation layer weights the output of the hidden neurons belonging to the same class in the hidden layer:

$$
v_{i}=\sum_{j=1}^{L} \phi_{i j} / L
$$

Where the vi represents the output of the $i$ category, and L represents the number of neurons in the $\mathrm{i}$ class. The number of neurons in the summation layer is the same as the number of classes $\mathrm{M}$.

The output layer fetches the largest one in the layer as the output of the category:

$$
y=\arg \max \left(v_{i}\right)
$$

The fault diagnosis method based PNN is an effective decision method which can be proved by probability statistic [12], which can be described as follows:

Suppose there are two known failure modes and, there is a set of fault feature samples to be judged:

If $h \operatorname{AlAfA}(X)>h B l B f B(X)$, so $X \in \theta A$

If $h A 1 A f A(X)<h B l B f B(X)$, so $X \in \theta B$

In the above formula, $\mathrm{hA}$ and $\mathrm{hB}$ are the priori probability for failure mode $\theta \mathrm{A}$ and $\theta \mathrm{B}(\mathrm{hA}=\mathrm{NA}$ $/ \mathrm{N}, \mathrm{hB}=\mathrm{NB} / \mathrm{N})$; $\mathrm{NA}$ and $\mathrm{NB}$ are the number of training samples for failure mode $\theta \mathrm{A}$ and $\theta \mathrm{B}$; $\mathrm{N}$ is total number of training samples; $1 \mathrm{~A}$ is the cost factor for erroneously dividing the fault feature sample $\mathrm{X}$ that should belong to $\theta \mathrm{A}$ to fault mode $\theta \mathrm{B} ; \mathrm{BB}$ is the cost factor for erroneously dividing the fault feature sample $X$ that should belong to $\theta B$ to fault mode $\theta A$; $f A(X)$ and $f B(X)$ are the probability density function for failure mode $\theta \mathrm{A}$ and $\theta \mathrm{B}$. Usually the probability density function cannot be obtained accurately, it only can get statistical value according to the existing fault feature samples.

\subsection{Extraction of Fault Data}

In the actual use of the lamination machine, the roller group may be tilted, or the roller group on the adhesion of foreign matter will lead to the quality of the lamination decline, so the roller group failure can be divided into four categories: the rollers are tilted to the left (Figure 7(a)), the rollers are tilted to the right (Figure 7 (b)), there are foreign bodies on the roller (Figure 7 (c)) and the rollers are normal (Figure 7 (d)).

(a)

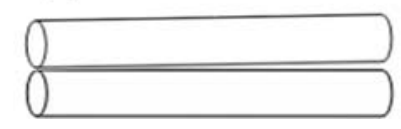

(b)



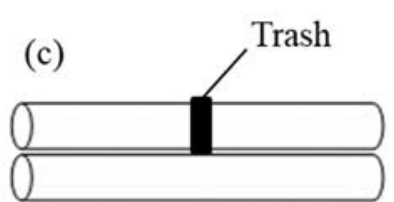

Fig. 7 Four fault modes schematic of roller group. (a) Left deviation of roller group. (b) Right deviation of roller group. (c) Exist trash on the roller group. (d) Normal of roller group.

According to the fault features, four man-made failure modes is shown in figure 7. (a) the rollers are tilted to the left; (b) the rollers are tilted to the right; (c) exist trash on the roller: an insulating tape is attached around the rollers on the upper side of the roller set; (d) the rollers are normal: remove the insulating tape and clean the upper and lower rollers. The thickness of the single-layer insulating tape is about $200 \mathrm{um}$ and the length of the roller set is about $38 \mathrm{~cm}$, and the inclination of the roller set is about $0.09^{\circ}$. Using the array sensor and data acquisition system, 400 data were collected at different positions of the roller group for four failure modes, and a data set containing 1600 data samples was obtained, each of which was a $16 \times 16$ matrix. The data sample is plotted as a pressure distribution, and the pressure profile for the four failure modes is shown in Figure 8. 



Fig. 8 Roller pressure distribution in four failure modes. (a) Left deviation of roller group. (b) Right deviation of roller group. (c) Exist trash on the roller group. (d) Normal of roller group.

\subsection{Fault Detection Steps}

The fault detection of roller group based on PNN mainly includes the training of fault detection model and the prediction of fault test data. The detailed steps as follows:

The data set is divided into training data sets and test data sets according to a certain proportion.

The training data set and the test data set are processed into a vector of length 256 and normalized.

Set the smoothing factor of PNN and train the PNN fault detection model;

The fault detection model is used to predict each data sample in the test data set.

Get forecast result.

\section{Test Experiment}

In order to prove the feasibility of the application of probabilistic neural network in the fault detection of roller group, we use MATLAB simulation experiment platform to experiment. $10 \%$ of the data samples are randomly selected from the data set as the test data, and the remaining data samples are used as training samples. We compare the fault detection model of PNN with the fault detection model of BP. Then define the fault category, as is shown in Table 1.

Table 1. Fault category definition

\begin{tabular}{cc}
\hline Fault category & Fault description \\
\hline 1 & Left deviation \\
2 & Normal \\
3 & Right deviation \\
4 & Exist trash \\
\hline
\end{tabular}

First, the data set is randomly divided into test data sets and training data sets according to the preset proportion and normalized.

Then, the PNN fault detection model and the BP neural network fault detection model are established by using MATLAB respectively:

netPNN = newpnn(trainx, ind2vec(trlab), spread);

netBP $=$ newff( $\operatorname{minmax}(\operatorname{trainx}),[804],\{$ 'tansig' 'tansig' $\}$, 'traingdx' $)$;

The default value of spread is 0.1 , after several tests, we found that the spread of PNN has the best results when the value is 1 . In the BP neural network, the number of input nodes is 256 , on a hidden layer, the number of nodes is 80 , there are four output layer nodes, the number of iterations is 2000 times, and the target error is 0.001 .

The test data sets were used to the two fault detection models for testing. The part of the predict results of PNN fault detection model is shown in Figure 9. The test results of the model construction training time and the prediction accuracy of the two fault detection models after twenty trials are shown in Table 2. 


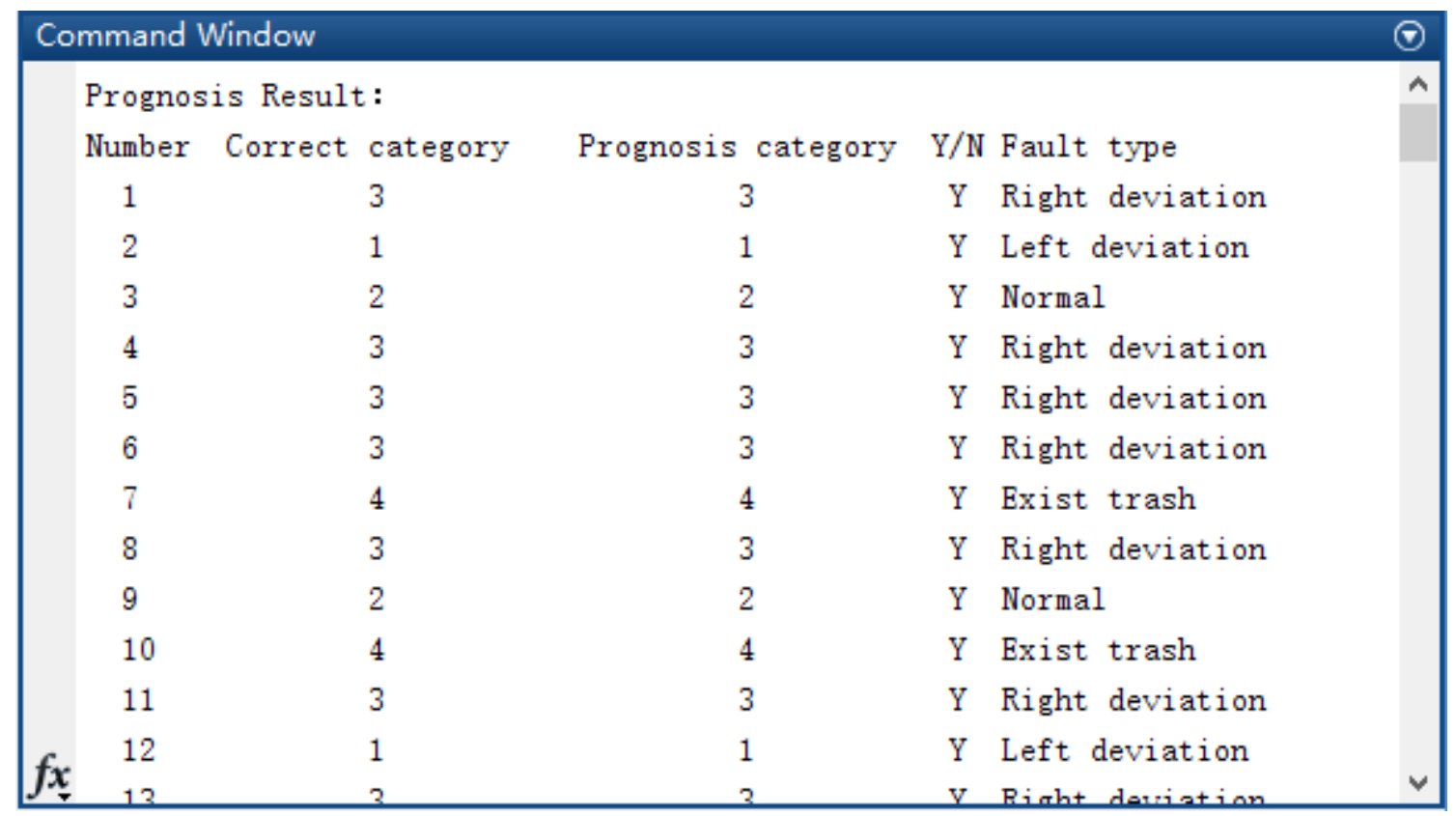

Fig. 9 Fault prediction results (PNN)

Table 2. Comparison of fault prediction results

\begin{tabular}{|c|c|c|c|c|}
\hline \multirow{2}{*}{ Test number } & \multicolumn{2}{|r|}{ BP } & \multicolumn{2}{|c|}{$\overline{\text { PNN }}$} \\
\hline & Time (s) & Correct rate (\%) & Time (s) & Correct rate (\%) \\
\hline 1 & 62.8 & 96.875 & 0.08 & 97.5 \\
\hline 2 & 57 & 98.125 & 0.079 & 98.75 \\
\hline 3 & 56 & 97.5 & 0.07 & 98.75 \\
\hline 4 & 49 & 96.25 & 0.083 & 99.375 \\
\hline 5 & 57 & 96.875 & 0.075 & 98.125 \\
\hline 6 & 60 & 97.5 & 0.086 & 98.125 \\
\hline 7 & 48 & 96.875 & 0.076 & 97.5 \\
\hline 8 & 61 & 98.75 & 0.085 & 100 \\
\hline 9 & 50 & 96.25 & 0.079 & 97.5 \\
\hline 10 & 45 & 97.5 & 0.074 & 98.125 \\
\hline 11 & 65 & 96.25 & 0.083 & 98.125 \\
\hline 12 & 62 & 97.5 & 0.085 & 99.375 \\
\hline 13 & 63 & 98.125 & 0.086 & 98.75 \\
\hline 14 & 62 & 99.375 & 0.091 & 99.375 \\
\hline 15 & 65 & 98.125 & 0.076 & 98.75 \\
\hline 16 & 58 & 97.5 & 0.077 & 98.125 \\
\hline 17 & 54 & 100 & 0.071 & 99.375 \\
\hline 18 & 55 & 96.875 & 0.086 & 97.5 \\
\hline 19 & 60 & 97.5 & 0.084 & 100 \\
\hline 20 & 61 & 98.125 & 0.075 & 98.75 \\
\hline Average & 57.54 & 97.59375 & 0.08005 & 98.59375 \\
\hline
\end{tabular}

After 20 experiments, the average accuracy rate of fault detection model based on probabilistic neural network is more than $98 \%$. It can be concluded that PNN fault detection model is superior to BP neural network fault both in model construction training time and in fault prediction accuracy Detection model. The Probabilistic neural network fault detection model is more suitable for detecting roll group failure. 


\section{Conclusion}

In this paper, a new array pressure sensor is designed, and this array is used to collect the pressure distribution data between the roller wheels. The pressure distribution data is processed by the probabilistic neural network to detect the fault of the roller group, and the fault neural network fault detection model is compared with the BP neural network fault detection model. It is proved that the probabilistic neural network fault detection model is more suitable for autonomous detection of roller group components. This method is more accurate and intuitive, it reduces the interference of environmental noise on fault detection to a large extent with breaking through the restrictions that the mechanical equipment, vibration, sound and other signals affect.

\section{Acknowledgments}

This work was supported by the National Natural Science Foundation of China (NSFC 61504148), the Basic Science and Frontier Technology Research Program of Chongqing (cstc2016jcyjA0315, cstc2017shmsA50001), Chongqing Collaborative Innovation Center for Brain Science, and Youth Innovation Promotion Association of CAS (2016340).

\section{References}

[1]. XIAO H J; HUANG D P. Fault diagnosis and prognosis of wastewater processes with incomplete data by the auto-associative neural networks and ARMA model [J]. Chemometrics and Intelligent Laboratory Systems, 2017, 161: 96-107.

[2]. LI Z N, ZHU M. Mechanical fault diagnosis method based on empirical wavelet transform [J]. Chinese Journal of Scientific Instrument, 2014, 34(11): 2423-2432.

[3]. XIANG D, CUI J. Method of roller bearing fault diagnosis based on feature fusion of EMD entropy [J]. Journal of Aerospace Power, 2015, 30(5): 1149-1155.

[4]. ZHANG J Y, ZHANG W. Intelligent Fault Diagnosis and Prediction for Equipment [M].Beijing: National Defense Industry Press, 2013.4.

[5]. YANG L X, ZHU Y L. High voltage circuit breaker fault diagnosis of probabilistic neural network [J]. Power System Protection and Control, 2015, 43(10): 62-67.

[6]. YANG M, CHEN H, LI M H. Brief Introduction to the Development of Flexible Array Pressure Sensor [J]. SPACECRAFT ENVIRONMENT ENGINEERING, 2009, 26: 112-115.

[7]. HUANG K K, ZHANG J, XIANG Z Y, et al. Tire ground analysis using a Tekscan pressure distribution measurement system [J]. Tire industry, 2008, 28(9): 563-565.

[8]. Haopeng Wang, Debao Zhou, and Jianguo Cao. Development of a Skin-Like Tactile Sensor Array for Curved Surface [J]. IEEE SENSORS JOURNAL, 2014, 14(1): 55-61.

[9]. LI D, HU X G. Redundant fault-tolerant system based on VxWorks and fault diagnosis of PNN [J]. Computer Engineering and Applications, 2016, 52(15): 13-18.

[10]. GAO Q H, WANG J G, SUN F L. PNN Recognition of License Plate Chinese Characters Based on Pseudo-Zernike Invariant Moments [J]. Computer Engineering, 2009, 35(4): 196-198.

[11]. CHEN M. MATLAB neural network theory and examples of fine solution [M].Beijing: TSINGHUA UNIVERSITY PRESS, 2013.3.

[12]. Yi J H, Wang J, Wang G G. Improved probabilistic neural networks with self-adaptive strategies for transformer fault diagnosis problem [J]. Advances in Mechanical Engineering, 2016, 8(1): 1-13. 